\title{
Co-administration of dextromethorphan with methamphetamine attenuates methamphetamine-induced rewarding and behavioral sensitization
}

\author{
Pao-Pao Yang ${ }^{1}$, Eagle Yi-Kung Huang ${ }^{1}$, Geng-Chang Yeh ${ }^{2} \&$ Pao-Luh Tao $^{1, *}$ \\ ${ }^{1}$ Department of Pharmacology, National Defense Medical Center, P.O. Box 90048-504, Nei-Hu 114, Taipei, \\ Taiwan, R.O.C; ${ }^{2}$ Department of Pediatrics, Taipei Medical University Hospital, Taipei Medical University, \\ Taipei, Taiwan, R.O.C
}

Received 26 April 2006; accepted 20 June 2006

(C) 2006 National Science Council, Taipei

Key words: behavioral sensitization, dextromethorphan, dopamine, methamphetamine, nucleus accumbens, rewarding, ventral tegmental area

\begin{abstract}
Summary
Methamphetamine (MA) is well known as a potent CNS stimulant, which produces strong rewarding and behavioral sensitization after repeated administration. In the present study, we investigated whether coadministration of dextromethorphan (DM) with MA could suppress these effects induced by acute and chronic MA treatment. The conditioned place preference (CPP) test was used to examine the rewarding/ drug seeking effects and locomotor and stereotypic activities were measured to investigate behavioral sensitization induced by chronic MA. Our results revealed that co-administration of DM (20 mg/kg, ip) with MA $(2 \mathrm{mg} / \mathrm{kg}$, ip) almost completely abolished the MA-induced CPP and behavioral sensitization. Furthermore, both of the acute and chronic MA could result in an increase of dopamine (DA) turnover rate in the NAc and mPFC. The acute effects of MA on DA turnover rate could be attenuated by the coadministration of DM in both regions. The chronic effect of MA on DA turnover rate in the mPFC was also attenuated by the co-administration of DM. These results suggest that the effect of DM on blocking MA-induced rewarding and behavioral sensitization may be related to its effect on inhibiting the activity of DA neurons projected to mPFC and/or NAc.
\end{abstract}

\section{Introduction}

The abuse of methamphetamine (MA) has increased considerably during the past two decades. It is thought to produce its reinforcing effects, at least in part, by activating the mesolimbic dopaminergic system [1, 2]. The dopamine (DA) projection from the ventral tegmental area (VTA) to the nucleus accumbens (NAc) and medial prefrontal cortex (mPFC) is thought to be involved in reward and in the regulation of cognitive and emotional behaviors [3]. This system also promotes behavioral sensitization, the result of chronic

*To whom correspondence should be addressed. Fax $+886-2$ 87923155, E-mail: pltao@mail.ndmctsgh.edu.tw exposure to psychostimulants $[4,5]$. Development of behavioral sensitization and subsequent cellular changes require transmission of glutamate, whose maintenance is linked to changes in glutamate receptor expression and sensitivity [6]. There is more and more evidence suggesting that $N$-methylD-aspartate (NMDA) receptors are involved in mediating the effects of substances of abuse [7-9]. However, those tested drugs (NMDA receptor antagonists: LY274614, CGP39551, MK-801) have not yet been proved to be clinically safe. Dextromethorphan (DM), a d-isomer of levorphanol, without any action on the opioid receptors, has been used as a regular antitussive drug with no apparent side effect [10]. DM can act as a noncompetitive antagonist on the NMDA receptor by 
binding to a site inside the NMDA receptor channel thereby blocks this channel [11]. Recent investigations had found that DM could effectively attenuate the tolerance and withdrawal syndrome of morphine in adult animals [12, 13]. In the previous study, we found that co-administration of DM with morphine has suppressing effects on the rewarding induced by morphine, and also on the increase of DA turnover induced by chronic morphine treatment [14]. In the present study, we further investigated whether co-administration of DM with MA can suppress the rewarding and behavioral sensitization induced by chronic MA. The DA turnover rates in the NAc and mPFC which were thought to be related to drug addiction were also determined.

\section{Methods}

\section{Animals}

Male Sprague-Dawley rats, weighing 280-400 g, were purchased from the National Experimental Animal Centre, Taipei, Taiwan, R.O.C. All rats were kept in an animal room with a $12 \mathrm{~h}$ light/ dark cycle, at a temperature of $25 \pm 2{ }^{\circ} \mathrm{C}$ and humidity of $55 \%$. Two or three animals were kept in a cage. Standard diet and water were provided ad libitum. The animals were acclimated for at least 1 week before the experiments. The care of animals was carried out in accordance with institutional and international standards (Principles of Laboratory Animal Care, NIH) and the protocol has got an approval from the Institutional Animal Care and Use Committee of National Defense Medical Center, Taiwan, R.O.C. There were at least eight animals in each experimental group.

\section{Schedule of drug administration}

As shown in Fig. 1, a total of 23-day schedule was used in this study. On day-1, the animals were placed in an isolated dark room (CPP test room) for $60 \mathrm{~min}$ for habituation. CPP conditionings and drug injections (MA $2 \mathrm{mg} / \mathrm{kg}$ or MA $2 \mathrm{mg} /$ $\mathrm{kg}+\mathrm{DM} 20 \mathrm{mg} / \mathrm{kg}$ ) were performed from day 3 to day 8 with saline injection (i.p.) in the morning and drug injection (i.p.) in the afternoon. For the control group, saline was injected instead of drug. CPP tests were carried out in the morning of day 1 ,

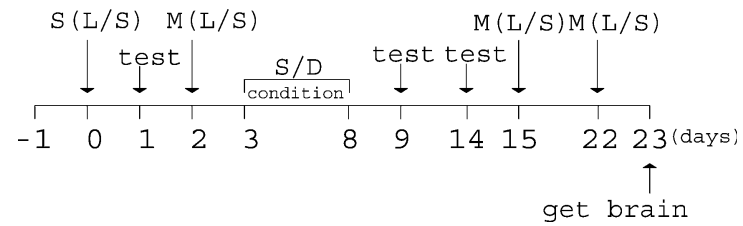

Figure 1. The schedule of experiment. The descriptions of the abbreviations in this figure are listed as the following: $\mathbf{M}$ : MA (2 mg/kg); D: MA (2 mg/kg) or MA $(2 \mathrm{mg} / \mathrm{kg})+$ DM; S: saline; test: CPP test; $\mathbf{L} / \mathbf{S}$ : locomotion and stereotypic activity.

day 9, and day 14. Locomotor activity and stereotypic activity tests were carried out in the afternoon on day 0 (after saline injection as control), day 2 (after MA or MA + DM injection), day 15 and day 22 (after MA or MA + DM injection). Rats were sacrificed by decapitation 20 min after MA or MA + DM challenge on day 23. Rat brains were removed, dissected and frozen by dry ice immediately, and then stored at $-80^{\circ} \mathrm{C}$ until used for the analysis of the DA turnover rate.

\section{Conditioned place preference test}

The CPP test apparatus, made from an acrylic plastic box $(70 \times 25 \times 25 \mathrm{~cm})$, was divided into three compartments. Two identically sized compartments $(30 \times 25 \times 25 \mathrm{~cm})$ were constructed at both sides, separated by a narrower compartment $(10 \times 25 \times 25 \mathrm{~cm})$. The compartments were connected by guillotine doors $(10 \times 10 \mathrm{~cm})$ in the central unit. One of the large compartments was covered by mosaic-type paper $(2.5 \times 2.5 \mathrm{~cm}$ black and white squares) on the three walls and floor, as a visual cue; the other large compartment was covered by purely white paper. To give more visual cues, a blue and a red light bulb were hung separately above the two large compartments. During the experiments, the CPP apparatus was kept in an isolated dark room, which was free from noise. After each behavioral test or place conditioning, the whole box was cleaned thoroughly to prevent the interference from the smell of feces and urine. For CPP conditionings, the rats were given saline in the morning and saline (control group) or MA or MA + DM in the afternoon for 6 days. A distinctive environment was paired repeatedly with administration of saline and a different environment was associated with MA injection. The animals were kept for $40 \mathrm{~min}$ in the corresponding compartment with the guillotine doors closed. 
CPP tests were performed on day 1 , day 9 and day 14 . We determined the place preference by placing the rats into the central compartment of the apparatus with the guillotine doors opened for $15 \mathrm{~min}$. The time that the rat stayed in each compartment was recorded to determine the place preference. The measurement of drug-seeking behavior was thought to be the increase in the time spent in the compartment previously paired with MA injection.

\section{Locomotor activity and stereotypic activity tests}

The ambulatory and the total activity of rats were measured in transparent standard polypropylene animal cages $(38 \times 22 \times 15 \mathrm{~cm})$. The test cages were placed in a 4-photobeam activity system (San Diego Instruments, USA) in an isolated noise-free room. A computer controlled unit recorded the interruption of photobeams from four individual cages. Ambulatory activity was recorded as breaks of two consecutive beams, whereas breaks of any single photobeam were determined as total activity. The activities were recorded in 5-min periods for $2 \mathrm{~h}$ immediately after saline or drug(s) administration. During each test session, each animal was also observed at 5-min intervals for their stereotypic activity. The quantitative scores of the stereotypic activity were assessed according to the following scale [15]:

0: No stereotypic activity. Generally, animals receiving this score were asleep.

1: Exploratory locomotion.

2: Sniffing (not intense) and some locomotor activity.

3: Erratic hyperactive locomotion and rearing; sniffing.

4: Running in circles; bouts of lying down and jumping up.

5: Gnawing; or extremely high levels of locomotion; rearing and jumping.

\section{Neurochemical measurements}

In this study, rats were divided into six groups ( $n=8$ for each group). Except three chronic groups as we described before (chronic control group, MA group and MA + DM group), we also included acute DM, acute MA, and acute
MA + DM group. Rats were sacrificed $20 \mathrm{~min}$ after saline (control group) or drug(s) administration and the brains were rapidly removed. The whole brain was placed in an acrylic brain matrix $(1 \mathrm{~mm}$ section coronal, Braintree Scientific, USA). Using a sharp blade, one third of the full length of brain (without the olfactory bulbs and the cerebellum) was vertically cut off from the rostral side. A sagittal slice of $1 \mathrm{~mm}$ was obtained by slicing at the adjacent indent of the matrix. Co-ordinating with the anterior commissure as a landmark, the NAc on the slice was carefully dissected out with a forceps. The mPFC was obtained from the previous slice, which was the horizontal layer above the NAc site. The dissected tissues were immediately frozen on top of dry ice and put into a $-80^{\circ} \mathrm{C}$ freezer. On the day of assay, the dissected tissue was homogenized in $0.1 \mathrm{mM}$ oxalic acid. The homogenates were centrifuged at $20,000 \mathrm{~g}$ for $40 \mathrm{~min}$ at $4^{\circ} \mathrm{C}$. The resulting supernatants were filtrated through a $0.22 \mu \mathrm{m}$ syringe filter (Millipore, Bedford, MA, USA) followed by high-performance liquid chromatography (HPLC) analysis. The HPLC system was composed of a reverse-phase C-18 column (MD-150, RP-C-18, $3 \mu \mathrm{m}$, length: $15 \mathrm{~cm}$, ESA, USA), a high-pressure pump (LC-10AD, Shimadzu, Japan), and connected with an electrochemical detector coupled with three electrodes (Coulochem II, ESA, USA). The electrode of the guard cell was set at $350 \mathrm{mV}$, whereas electrode 1 and electrode 2 (for detection) were set at 40 and $250 \mathrm{mV}$, respectively. Under an isocratic condition, the commercial solvent of mobile phase (MD-TM, 70-1332; ESA, USA) consisting of $75 \mathrm{mM} \mathrm{NaH} \mathrm{PO}_{4}, 30 \mathrm{mM}$ citric acid, $0.1 \mathrm{mM}$ EDTA, $0.02 \%$ sodium octylsulfate, and $9 \%$ methanol ( $\mathrm{pH} 3.3-3.5)$ was pumped and circulated at a flow rate of $0.5 \mathrm{ml} / \mathrm{min}$ in the system. The concentrations of DA, 3, 4-dihydroxyphenylacetic acid (DOPAC) and homovanillic acid (HVA) were determined with the electrochemical detector. To quantify the sample peak, each chemical was compared with the external standards, which were freshly prepared and injected every five sample runs.

\section{Statistical analysis}

The data were expressed as the mean \pm SEM. Analysis of variance was used to access the 
statistical significance for repeated measures of the data, and the differences between the individual mean values in different groups were analyzed by ANOVA followed by Newman-Keuls test. In the CPP test, the difference of place preference in the same animal was analyzed by paired $t$ test. The differences were considered to be significant at $p<0.05$ or $p<0.01$.

\section{Chemicals}

Methamphetamine hydrochloride was purchased from the National Bureau of Controlled Drugs, National Health Administration, Taipei, Taiwan, R.O.C. Citric acid, EDTA and methanol were supplied by J.T. Baker Chemical Inc. (Phillipsburg, $\mathrm{NJ}$, USA). Oxalic acid and $\mathrm{NaH}_{2} \mathrm{PO}_{4}$ were supplied by Nacalai Tesque Inc. (Kyoto, Japan). The other chemicals were all purchased from Sigma (St. Louis, MO, USA). The chemicals were all of analytical grade.

\section{Results}

Effect of co-administration of DM on the rewarding effect induced by $M A$

The conditioned place preference (CPP) test was used to measure the rewarding effect of tested $\operatorname{drug}(\mathrm{s})$. The time spent in the drug-paired compartment minus the time spent in the saline-paired compartment was expressed as the place preference induced by drug(s) (Figure 2). In the control group (saline was injected as the drug), there was no significant place preference for the drug-paired compartment on day $1(30.5 \pm 33.6 \mathrm{~s})$, indicating that the CPP apparatus provided an unbiased design [16]. There was also no significant place preference on day $9(9.0 \pm 67.9 \mathrm{~s})$ or day 14 $(-36.8 \pm 155.3 \mathrm{~s})$ (Fig. 2a). Administration of MA (2 mg/kg, i.p.) and conditioning for 6 days markedly increased $(p<0.01)$ the time spent in the MA-paired compartment $(431.1 \pm 104.2 \mathrm{~s})$ on day 9 as shown in Fig. 2b, indicating that MA had a significant rewarding effect. After 6 days of withdrawal from MA, rats still showed a significant place preference $(287.5 \pm 81.9 \mathrm{~s})$ for the MA-paired compartment on day 14, suggesting that the drug-seeking effect of MA remained. When DM was co-administered with MA, the
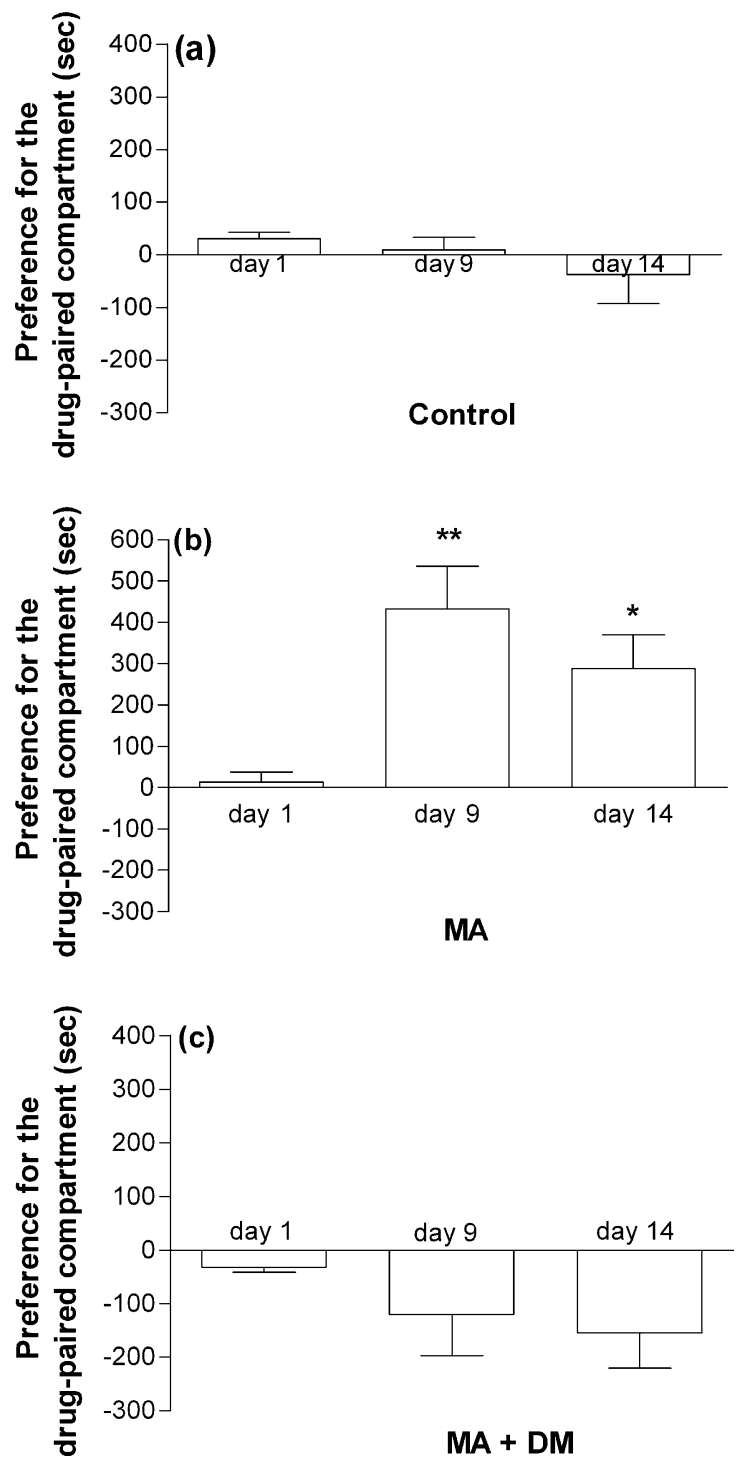

Figure 2. Effect of co-administration of DM with MA on the rewarding and drug-seeking effects measured by conditioned place preference (CPP) test. (a) Control group (saline, i.p.) (b) MA group (MA, $2 \mathrm{mg} / \mathrm{kg}$, i.p.) (c) MA (2 mg/kg, i.p.) + DM $(20 \mathrm{mg} / \mathrm{kg}$, i.p.) group. Data were expressed as the preference for the drug-paired compartment as determined by time spent in the drug-paired compartment minus time spent in the saline-paired compartment. Data were presented as mean \pm S.E.M. $(n=8)$. Paired $t$-test was used to analyze the data. Data on day 9 or day 14 of the same animal was compared to its data on day $1\left(^{*} p<0.05,{ }^{* *} p<0.01\right.$ vs. day 1).

rewarding and drug-seeking effects of MA were completely abolished on day $9(-119.9 \pm 76.9 \mathrm{~s})$ and day $14(-155.1 \pm 64.9 \mathrm{~s}$ ) (Figure 2c) and showed a tendency of aversion but without statistic significance. 


\section{Chronic MA-induced behavioral sensitization}

Acute MA treatment significantly increased the ambulatory and total activity of animals (Table 1). Stereotypic score after acute MA treatment on day 2 was also much higher than those of saline-treated rats on day $0(p<0.01)$ (Table 1). After 6 days of chronic MA treatment $(2 \mathrm{mg} / \mathrm{kg}$, i.p. per day) and 6 days of withdrawal from MA (day 9 to day 14), the ambulatory activity and total activity induced by a single injection of MA ( $2 \mathrm{mg} / \mathrm{kg}$, i.p.) on day 15 were also significantly higher than those recorded on day $0(p<0.01)$ but not higher than those recorded on day 2 (Table 1). However, the stereotypic score was significantly higher than those recorded following acute MA on day 2 . After further withdrawal of MA for 6 days (day 16 to day 21), the ambulatory activity, total activity and stereotypic score induced by MA $(2 \mathrm{mg} / \mathrm{kg}$, i.p.) on day 22 were all significantly higher than those on day 0 (saline, $p<0.01$ ) or day 2 (acute MA, $p<0.05$ or $p<0.01$ ) (Table 1). These results indicated that the animals have developed behavioral sensitization to MA on day 22 under this protocol.

\section{Effect of co-administration of DM on chronic MA-induced behavioral sensitization}

In a different group of rats, acute MA+DM $(2 \mathrm{mg} / \mathrm{kg}$ of $\mathrm{MA}$ and $20 \mathrm{mg} / \mathrm{kg}$ of DM) also increased the ambulatory activity, total activity and stereotypic score (Table 2) as MA did (Table 1). After 6 days of chronic MA + DM injection and 6 days withdrawal of MA + DM (day 9 to day 14), the ambulatory activity and total activity induced by a single injection of $\mathrm{MA}+\mathrm{DM}$ on day 15 were significant higher than those on day $0(p<0.01)$ but not higher than that of acute MA + DM on day 2 (Table 2). The stereotypic scores were also significantly higher than those recorded on day 0 (saline), but they were significantly lower than those of acute MA + DM on day $2(p<0.01)$. After further withdrawal of MA + DM for 6 days (day 16 to day 21 ), the ambulatory activity and total activity induced by MA + DM on day 22 were significant higher than those on day 0 $(p<0.01)$ but not higher than those on day 2 (Table 2). The stereotypic scores were not higher but significantly lower than that of acute $\mathrm{MA}+\mathrm{DM}$ on day 2. These results indicated that chronically co-administration of DM with MA inhibited the behavioral sensitization induced by MA under this protocol.

Effect of co-administration of DM with MA on the $D A$ turnover rate in various brain regions

Figure 3 shows that acute MA injection $(2 \mathrm{mg} /$ $\mathrm{kg}$, i.p.) significantly increased the DA turnover rate in the NAc and mPFC. Acutely co-administration of DM with MA reversed the effect of MA in the NAc and mPFC, although acute DM by itself did not affect the basal DA turnover rate. After chronic MA treatment and withdrawal for about 2 weeks (except injection of drug on day 15 and day 22 for determination of locomotor activities and stereotypic score), a single injection of MA on day 23 still increased the DA turnover rate in the NAc $(p<0.05)$ and $\operatorname{mPFC}(p<0.01)$. Chronically co-administration of DM with MA significantly reversed the increase of the DA turnover rate induced by chronic MA in the mPFC but not in the NAc (Figure 3).

Table 1. Effect of chronic MA (2 mg/kg, i.p.) on behavioral sensitization.

\begin{tabular}{lccc}
\hline & \multicolumn{2}{l}{ Locomotor activity } & \multirow{2}{*}{ Stereotypic scores } \\
\cline { 2 - 3 } & Ambulatory & Total & \\
\hline Control (Day 0) & $69.1 \pm 15.5$ & $536.0 \pm 89.0$ & $10.8 \pm 2.1$ \\
Acute MA (Day 2) & $1163.4 \pm 131.5^{* *}$ & $2519.9 \pm 206.6^{* *}$ & $65.6 \pm 1.2^{* *}$ \\
Chronic MA (Day 15) & $1277.9 \pm 161.1^{* *}$ & $2924.1 \pm 269.2^{* *}$ & $71.9 \pm 0.9^{* * \# \#}$ \\
Chronic MA (Day 22) & $1519.1 \pm 58.5^{* *}$ & $3189.8 \pm 150.6^{* * \#}$ & $74.45 \pm 0.5^{* * \# \#}$ \\
\hline
\end{tabular}

Data are presented as means \pm S.E.M. $(n=8)$. One way ANOVA and Newman-Keuls test were used to analyze the data $\left({ }^{* *} p<0.01\right.$ vs. saline; ${ }^{\#} p<0.05,{ }^{\# \#} p<0.01$ vs. day 2$)$. 
Table 2. Effect of co-administration of DM (20 mg/kg, i.p.) on behavioral sensitization induced by chronic MA $(2 \mathrm{mg} / \mathrm{kg}$, i.p.) treatment.

\begin{tabular}{lccc}
\hline & \multicolumn{2}{c}{ Locomotor activity } & \multirow{2}{*}{ Stereotypic scores } \\
\cline { 2 - 3 } & Ambulatory & Total & \\
\hline Control (day 0) & $69.0 \pm 10.0$ & $469.0 \pm 107.9$ & $8.6 \pm 4.0$ \\
Acute (MA + DM) (day 2) & $1344.6 \pm 147.6^{* *}$ & $2917.4 \pm 245.7^{* *}$ & $8.4 \pm 1.1^{* *}$ \\
Chronic (MA + DM) (day 15) & $1345.3 \pm 158.6^{* *}$ & $3299.3 \pm 294.2^{* *}$ & $61.9 \pm 2.5^{* * \# \#}$ \\
Chronic (MA + DM) (day 22) & $1355.9 \pm 176.1^{* *}$ & $3009.1 \pm 258.8^{* *}$ & $57.3 \pm 1.5^{* * \# \#}$ \\
\hline
\end{tabular}

Data are presented as means \pm S.E.M. $(n=8)$. One way ANOVA and Newman-Keuls test were used to analyze the data $\left({ }^{* *} p<0.01\right.$ vs. control; ${ }^{\#} p<0.01$ vs. day 2$)$.

\section{Discussion}

In the CPP test of this study, we found that MA induced a significant place preference for the drugpaired compartment on day 9 and day 14. This indicated that chronic MA treatment did cause rewarding and drug-seeking effects under our protocol. Co-administration of DM with MA significantly reversed these effects and showed a tendency of aversion (Figure 2). Behavioral sensitization has also been shown in chronic MA group on day 15 (stereotypic activity) or day 22 (locomotor activity and stereotypic activity) (Table 1). Co-administration of DM with MA significantly suppressed the behavioral sensitization in both locomotor activity and stereotypic activity induced by chronic MA (Table 2). In our previous report, we found that DM by itself had no rewarding or aversive effect [14]. The possible outcome of aversion if produced by co-administration of DM with MA could decrease the incentive of addicts. This is similar as using disulfiram to treat alcohol addicts. Disulfiram given by itself to nondrinkers has little effect; however, flushing, headache, nausea, vomiting, etc. occur within a few minutes after drinking alcohol. Therefore the aversion produced by disulfiram with alcohol decrease the incentive of addicts to drink alcohol. Similar thing may happen in MA addicts to use DM with MA together. Therefore this property of DM may be useful to help MA addicts to quit MA.

Behavioral sensitization is presumed to be mediated by the mesolimbic dopaminergic system [17, 18], which is the same neuronal system thought to be mediating the rewarding effect. Since the DA turnover rate may represent the dopaminergic neuronal activity, we have deter- mined the DA turnover rate in two brain regions (NAc and mPFC) which are usually considered to be related to drug addiction [19]. We found that acute MA treatment increased the DA turnover rate in both brain regions. Co-administration of DM with MA acutely suppressed the DA turnover rate induced by MA in the NAc and the mPFC indicating that the DA neurons activated by MA in these regions may be partially through the activation of NMDA receptors. When DM was co-administered with MA, it might decrease the effect of MA on DA release through blocking the NMDA receptors. Chronic MA treatment still increased the DA turnover rate in both the NAc and the mPFC. However, chronic co-administration of DM with MA only significantly suppressed the DA turnover rate in the mPFC.

In 1997, Kim and Jang found that a noncompetitive antagonist of NMDA receptors, dizocilpine (MK-801), could significantly attenuate the place preference induced by MA when administered (i.p.) in animals in the CPP tests [20]. In this report, they also showed that CNQX (the antagonist of AMPA/kainate receptors) could attenuate the place preference induced by MA. In addition, Jhoo et al. reported that DM at the dose of $(20 \mathrm{mg} / \mathrm{kg}$, i.p.) could abolish the place preference induced by cocaine $(2.5,5,10$ and $20 \mathrm{mg} / \mathrm{kg}$, i.p.)[21]. These reports are consistent with the finding in our study. From the experimental results of the present study, it was demonstrated that DM was able to abolish the place preference induced by MA ( $2 \mathrm{mg} / \mathrm{kg})$, when co-administered with MA in the CPP tests. Thus, it may also imply that DM can decrease the drug-seeking behavior caused by chronic MA treatment.

Riluzole, a glutamate release inhibitor, was shown to suppress the behavioral sensitization 


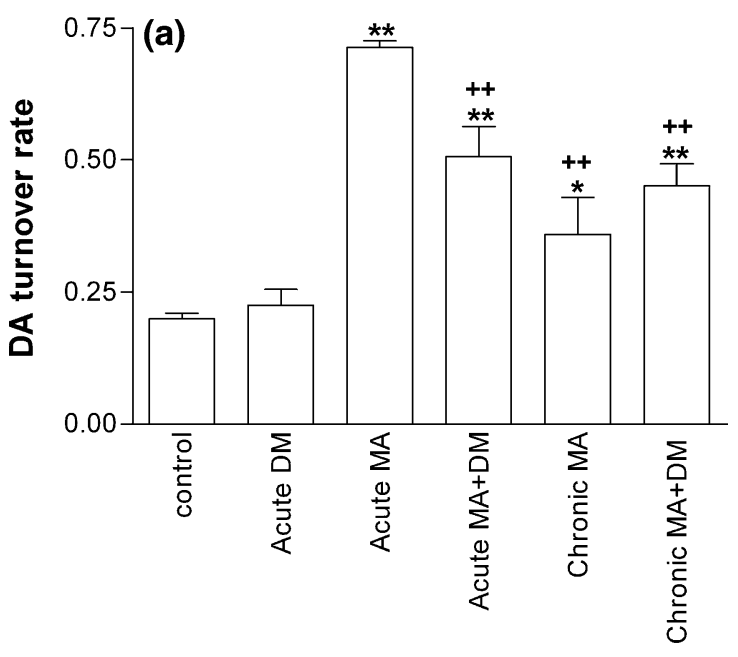

NAC

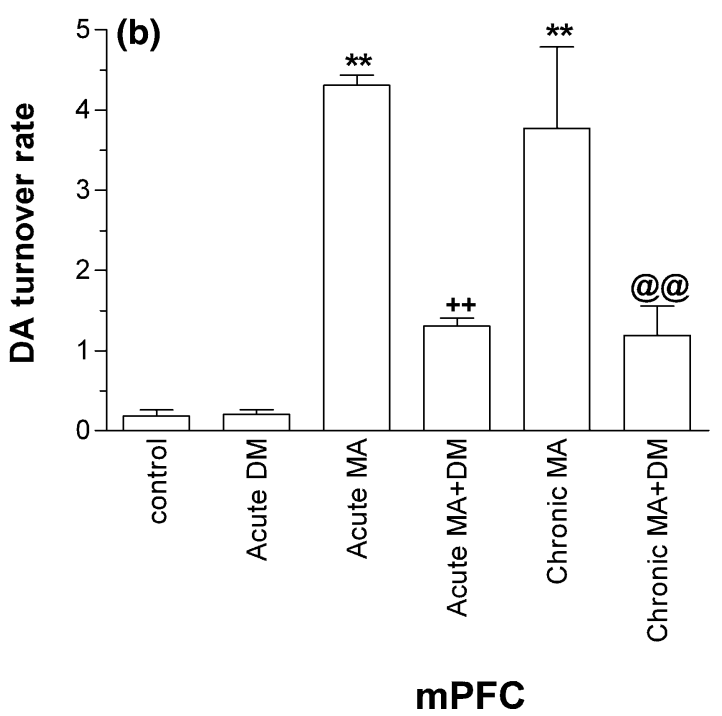

Figure 3. Effect of co-administration of DM with MA (2 mg/ $\mathrm{kg}$ ) on the dopamine turnover rate $[(\mathrm{DOPAC}+\mathrm{HVA}) / \mathrm{DA}]$ in (a) nucleus accumbens (NAc) (b) medial prefrontal cortex (mPFC) $(n=6-8)$. Data are presented as means \pm S.E.M. One way ANOVA and Newman-Keuls test were used to analyze the data $\left(^{*} p<0.05 ;{ }^{* *} p<0.01\right.$ vs. control group; ${ }^{++} p<0.01$ vs. acute MA group; ${ }^{@}{ }^{@} p<0.01$ vs. chronic MA group).

induced by MA [22]. Therefore, it was speculated that the excitatory amino acids may play an important role in the expression of behavioral sensitization induced by MA. However, Ujike et al. reported that both the competitive NMDA receptor antagonist, CPP ([3- $(+/-)$-2-carboxypiperatzin-4yl]-propyl-1-phosphonic acid), and the non-competitive NMDA receptor antagonist,
MK-801, were unable to inhibit the behavioral sensitization caused by MA [23]. Therefore, it should be noticed that the behavioral sensitization caused by addictive drugs could be attributed to some NMDA receptor-independent mechanisms. As to the stereotypic behavior induced by MA, NBQX (glutamatergic AMPA receptor antagonist) could not inhibit the increased stereotypic activity caused by chronic MA administration [24], but MK-801 (non-competitive NMDA receptor antagonist) could decrease the stereotypic activity [25]. In the present study, we found that DM could inhibit the behavioral sensitization of locomotor activity induced by MA administration, as well as that of the stereotypic activity.

In summary, we found that co-administration of DM with MA attenuates MA-induced rewarding and behavioral sensitization. In our previous study, we reported that co-administration of DM with morphine attenuates morphine rewarding effect and related DA releases at the nucleus accumbens [14]. The present results further extend the potential application of DM and confirm the profound negative effects of DM, possibly via the blockade of NMDA receptors, on the dopaminergic mesolimbic pathway in correlation with rewarding and behavioral sensitization.

\section{Acknowledgment}

This study was supported by the grants from National Science Council (NSC 93-2320-B-016033), Taipei, Taiwan, Republic of China.

\section{References}

1. Hoffman D.C., The use of place conditioning in studying the neuropharmacology of drug reinforcement. Brain Res. Bull. 23: 373-387, 1989.

2. Swerdlow N.R., Vaccarino F.J., Amalric M. and Koob G.F., The neural substrates for the motor-activating properties of psychostimulants: a review of recent findings. Pharmacol. Biochem. Behav. 25: 233-248, 1986.

3. Simon H., Scatton B. and Moal M.L., Dopaminergic A10 neurones are involved in cognitive functions. Nature 286: 150-151, 1980.

4. Kalivas P.W. and Stewart J., Dopamine transmission in the initiation and expression of drug- and stress-induced sensitization of motor activity. Brain Res. Brain Res. Rev. 16: 223-244, 1991.

5. Strakowski S.M. and Sax K.W., Progressive behavioral response to repeated D-amphetamine challenge: further 
evidence for sensitization in humans. Biol. Psych. 44: 11711177, 1998

6. Wolf M.E., The role of excitatory amino acids in behavioral sensitization to psychomotor stimulants. Prog. Neurobiol. 54: 679-720, 1998.

7. Tiseo P.J., Cheng J., Pasternak G.W. and Inturrisi C.E., Modulation of morphine tolerance by the competitive $\mathrm{N}$ methyl-D-aspartate receptor antagonist LY274614: assessment of opioid receptor changes. J Pharmacol Exp Ther. 268: 195-201, 1994.

8. Trujillo K.A. and Akil H., Excitatory amino acids and drugs of abuse: a role for $N$-methyl-D-aspartate receptors in drug tolerance, sensitization and physical dependence. Drug Alcohol Depend. 38: 139-154, 1995.

9. Tokuyama S., Wakabayashi H. and Ho I.K., Direct evidence for a role of glutamate in the expression of the opioid withdrawal syndrome. Eur. J. Pharmacol. 295: 123129, 1996.

10. Bem J.L. and Peck R., Dextromethorphan. An overview of safety issues. Drug Saf. 7: 190-199, 1992.

11. Church J., Sawyer D. and McLarnon J.G., Interactions of dextromethorphan with the N-methyl-D-aspartate receptor-channel complex: single channel recordings. Brain Res. 666: 189-194, 1994.

12. Farzin D., Modification of naloxone-induced withdrawal signs by dextromethorphan in morphine-dependent mice. Eur. J. Pharmacol. 377: 35-42, 1999.

13. Mao J., Price D.D., Caruso F.S. and Mayer D.J., Oral administration of dextromethorphan prevents the development of morphine tolerance and dependence in rats. Pain 67: 361-368, 1996.

14. Huang E.Y., Liu T.C. and Tao P.L., Co-administration of dextromethorphan with morphine attenuates morphine rewarding effect and related dopamine releases at the nucleus accumbens. Naunyn Schmiedebergs Arch. Pharmacol. 368: 386-392, 2003.

15. Ranaldi R., Munn E., Neklesa T. and Wise R.A. Morphine and amphetamine sensitization in rats demonstrated under moderate- and high-dose NMDA receptor blockade with MK-801 (dizocilpine). Psychopharmacology (Berl) 151: 192-201, 2000.
16. Tzschentke T.M., Measuring reward with the conditioned place preference paradigm: a comprehensive review of drug effects, recent progress and new issues. Prog. Neurobiol. 56: 613-672, 1998.

17. Joyce E.M. and Iversen S.D., The effect of morphine applied locally to mesencephalic dopamine cell bodies on spontaneous motor activity in the rat. Neurosci. Lett. 14: 207-212, 1979.

18. Vezina P. and Stewart J., Conditioning and place-specific sensitization of increases in activity induced by morphine in the VTA. Pharmacol. Biochem. Behav. 20: 925-934, 1984.

19. Kelly P.H., Seviour P.W. and Iversen S.D., Amphetamine and apomorphine responses in the rat following 6-OHDA lesions of the nucleus accumbens septi and corpus striatum. Brain Res. 94: 507-522, 1975.

20. Kim H.S. and Jang C.G., MK-801 inhibits methamphetamine-induced conditioned place preference and behavioral sensitization to apomorphine in mice. Brain Res. Bull. 44: 221-227, 1997.

21. Jhoo W.K., Shin E.J., Lee Y.H., Cheon M.A., Oh K.W., Kang S.Y., Lee C., Yi B.C. and Kim H.C., Dual effects of dextromethorphan on cocaine-induced conditioned place preference in mice. Neurosci. Lett. 288: 76-80, 2000.

22. Itzhak Y. and Martin J.L., Effect of riluzole and gabapentin on cocaine- and methamphetamine-induced behavioral sensitization in mice. Psychopharmacology (Berl) 151: 226233,2000

23. Ujike H., Tsuchida H., Kanzaki A., Akiyama K. and Otsuki S., Competitive and non-competitive $N$-methyl-Daspartate antagonists fail to prevent the induction of methamphetamine-induced sensitization. Life Sci. 50: 1673-1681, 1992.

24. Li Y., Vartanian A.J., White F.J., Xue C.J. and Wolf M.E., Effects of the AMPA receptor antagonist NBQX on the development and expression of behavioral sensitization to cocaine and amphetamine. Psychopharmacology (Berl) 134: 266-276, 1997.

25. Segal D.S., Kuczenski R. and Florin S.M., Does dizocilpine (MK-801) selectively block the enhanced responsiveness to repeated amphetamine administration?. Behav. Neurosci. 109: 532-546, 1995. 